\title{
An innovative laparoscopic and endoscopic technique in an animal model: combined gastric full-thickness tumor resection
}

\section{(9) $\odot \ominus$}

\author{
Authors \\ Flavio Hiroshi Ananias Morita ${ }^{1}$, Christiano Makoto Sakai ${ }^{1}$, Flavio Masato Kawamoto ${ }^{1}$, Eduardo Guimarães Hourneaux \\ de Moura' ${ }^{1}$, Paulo Sakai ${ }^{1}$
}

Institution

University of São Paulo Medical School - Gastroenterology Department, Sao Paulo, Brazil

submitted 5.2.2018

accepted after revision 30.5.2018

Bibliography

DOI https://doi.org/10.1055/a-0658-1283 |

Endoscopy International Open 2019; 07: E440-E445

(c) Georg Thieme Verlag KG Stuttgart · New York

ISSN 2364-3722

Corresponding author

Flavio H. A. Morita, MD, Av. Doutor.Eneas de Carvalho

Aguiar, 255 - 6th Floor, Ambulatory Building H.C.-

Endoscopy Unit, 05403-000 São Paulo, SP, Brazil

Fax: +551126616467

flaviomorita@hotmail.com

\section{ABSTRACT}

Background and study aims The combination of endoscopy with laparoscopy for full-thickness gastric resection has received much attention. The advantage in using it is removak of the target lesion without resection of excessive normal tissue. The technique could prevent deformed scars, particularly at the cardia and in the prepyloric area. The aim of this protocol was to evaluate a new combined operation for full-thickness resection of the gastric wall.

Materials and methods Gastric subepithelial lesions in multiple topographic locations of the stomach were simulated in seven live pigs. Full-thickness gastric resection was undertaken and after assessment of the outcome, the animals were euthanized. The primary endpoint was accomplishment of the procedure following all steps in the new technique, in various gastric locations. The secondary endpoints were duration of the procedure, quality of specimen margins, and complications.

Results Resections were successful, complete, and complication-free, ensuring a safe surgical margin of healthy tissue. The procedure was completed in 50 minutes. No mucosal perforation or gas escape occurred.

Conclusions The combined technique was safe, effective and minimally invasive. No expensive materials were used. Lesion exposure, gastric content leakage, incomplete resection, and excessive normal tissue elimination were avoided. Human trials of this technique may be warranted.

\section{Introduction}

The combination of endoscopy with laparoscopy for full-thickness gastric resection of both benign and malignant lesions has received much attention [1,2]. Since 2008 , as originally described by Hiki et al [3], an array of minimally invasive interventions has been recommended. The advantage of the technique is the ability to surgically ablate a target lesion without removing excessive normal tissue. Precise resection and lack of mutilation also prevents deformed scars, particularly at the cardia and in the prepyloric area, which would result in functional impairment [2].

Indications for use of the technique focus on lesions not requiring lymphadenectomy, such as subepithelial, with especial emphasis on stromal (GIST) and neuroendocrine tumors, and early gastric adenocarcinoma. Sentinel nodes can be searched, aiming to elucidate the need for lymphadenectomy [4].

In a retrospective study [5], after standardization of laparoscopic and endoscopic cooperative surgery (LECS), operative blood loss, hospitalization length, and need for gastrectomy significantly diminished. An important role of LECS for tumors in the upper third of the stomach was envisaged. Maximal free resection margin was reduced, in comparison with surgical wedge resection.

The ideal procedure should, of course, be minimally invasive and also easy to perform, affordable, and applicable to most lesions. Other desirable features are safety and ability to resect a tumor without exposure, excessive margins, or spillage of gastric contents into the peritoneal cavity [6].

The primary endpoint of this protocol was accomplishment of the procedure in various gastric locations following all steps 
in the new technique. The secondary endpoints were duration of the procedure, quality of specimen margins (complete vs. incomplete removal), and incidence of complications (perforation, gas leakage).

\section{Materials and methods}

This study was conducted at the Experimental Surgical Unit of São Paulo University Medical School. Ethical Approval was obtained and all interventions followed the guidelines of the Institutional Animal Care and Use Committee, as well as the Brazilian and international legislation concerning the use and handling of laboratory animals.

\section{Animals}

Yorkshire pigs $(30 \mathrm{~kg}$ ) were submitted to general anesthesia, based on $2 \%$ sevoflurane and propofol $10 \mathrm{mg} / \mathrm{kg} / \mathrm{h}$. Premedication consisted of intramuscular ketamine $20 \mathrm{mg} / \mathrm{kg}$ and atropine $0.04 \mathrm{mg} / \mathrm{kg}$. Animals were euthanized at the end of the experiment by potassium chloride overdose.

\section{Anatomical sites}

Four lesions were simulated in the antrum (anterior and posterior wall, lesser and greater curvature), one in mid-corpus (lesser curvature), and two more at the cardia (anterior wall and lesser curvature). These locations were selected because they are among the most challenging for classic surgical wedge resection, on account of potential retractions and deformities. Antrectomy, subtotal gastrectomy, and even total gastrectomy, could be necessary without such combined surgery.

\section{Lesion modeling}

After selection of the sites, laparoscopy was performed. Given the large volume of the pig liver, the gallbladder was sutured to the anterior abdominal wall with a single stitch to improve gastric visibility.

Subepithelial tumors were simulated by means of implantation of an oval-shaped foreign body, namely a latex glove finger-tip, filled with a small gauze fragment (approximately $2 \mathrm{~cm}$ diameter). To prevent accidental opening of the mucosa, the submucosa was endoscopically injected with a $6 \%$ hydroxyethyl starch/indigocarmine staining solution. Afterwards an " $X$ " opening of the seromuscular layer was laparoscopically provided, allowing visualization of the stained submucosa. A small pocket was made by dissecting the submucosa and the foreign body was implanted, thus generating the "tumor." The seromuscular opening was then sutured to restore gastric integrity ( Fig. 1).
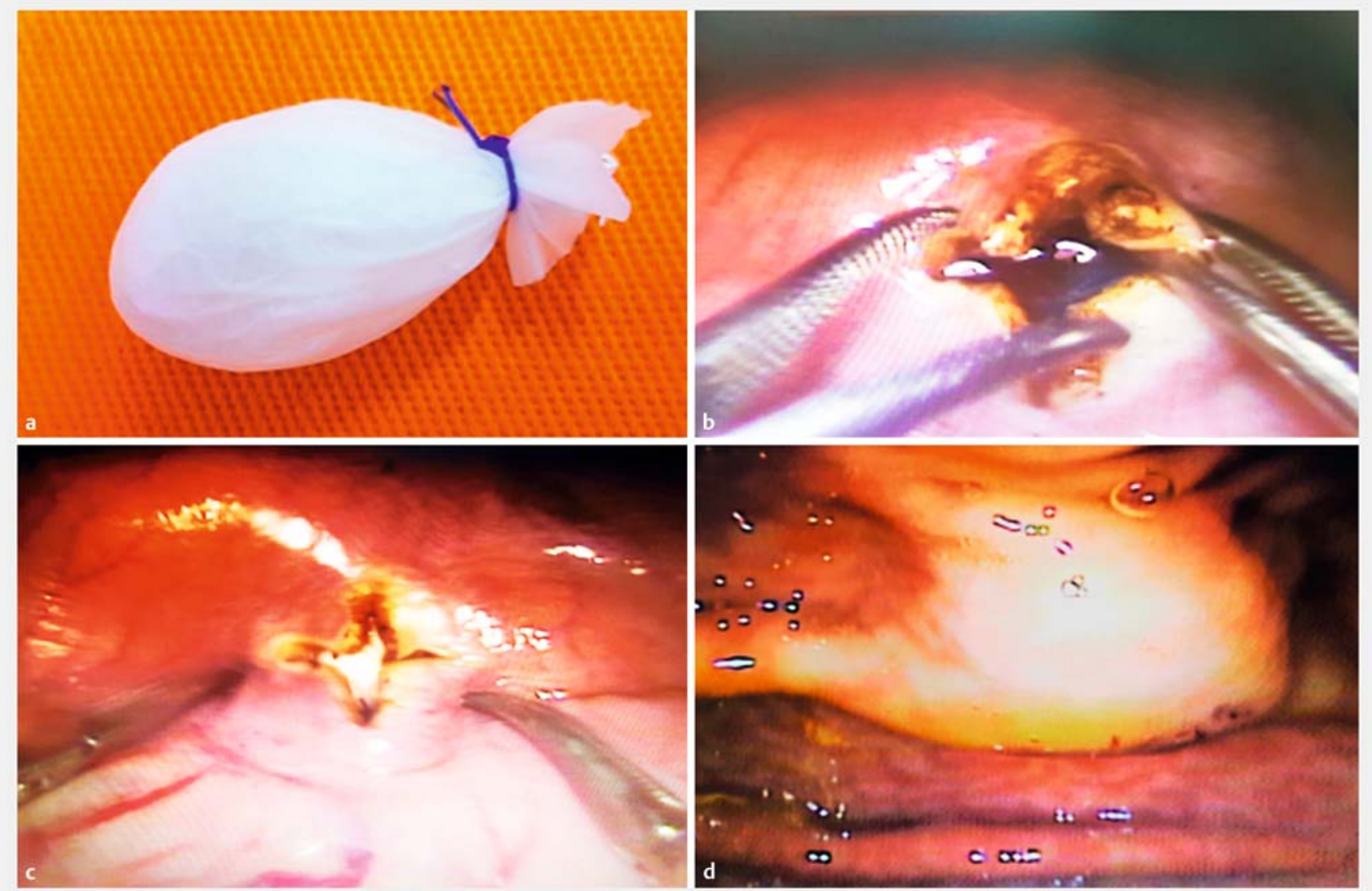

- Fig. 1 a Foreign body mimetizing the subepithelial lesion. b. Pocket dissected in the submucosa. c Foreign body in the submucosa, as seen from the seromuscular opening. $\mathbf{d}$ Endoscopic view of the simulated tumor. 


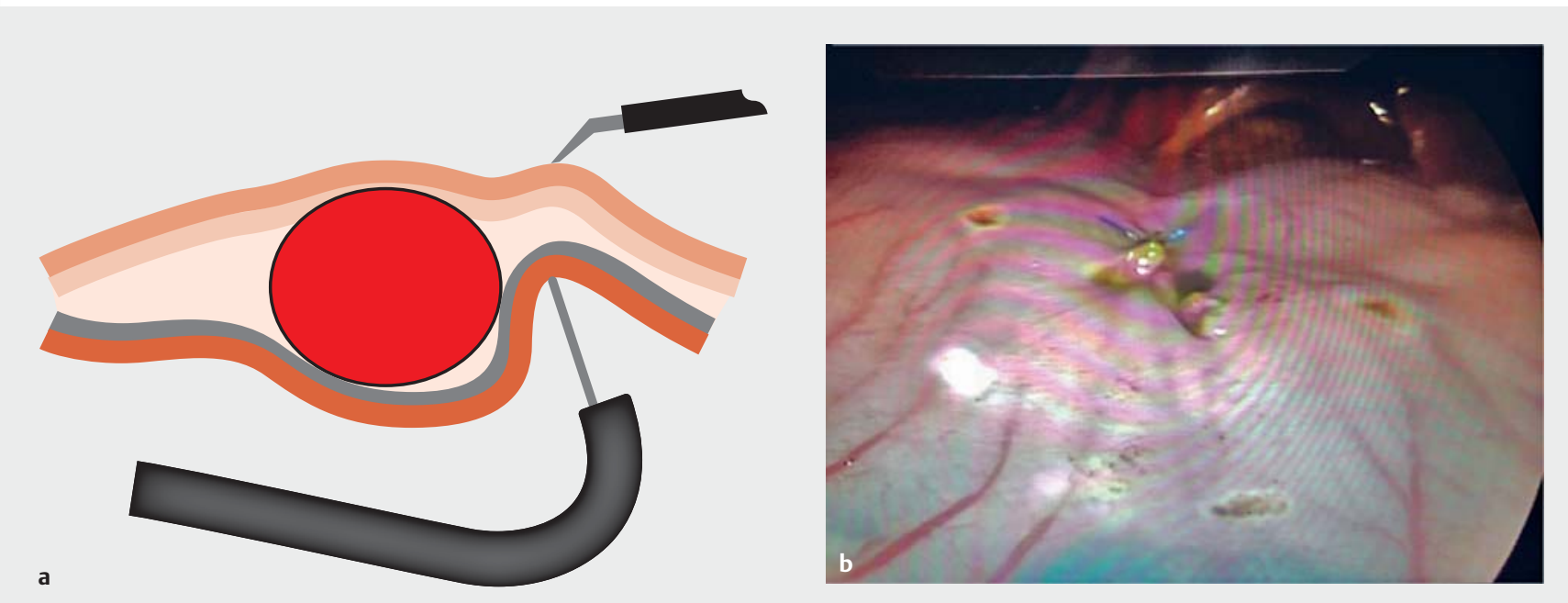

Fig. 2 Marking of the resection limits.
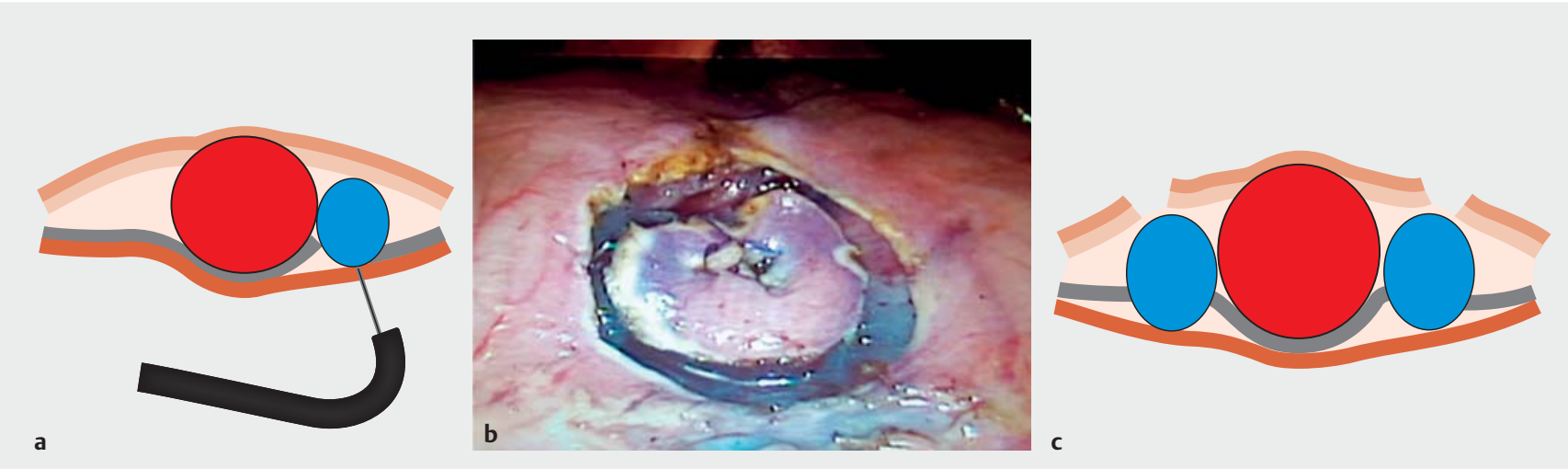

Fig. 3 Submucosal injection around the lesion, and incision of the seromuscular layer.

\section{Resection technique}

In the supine animal, the surgeon worked between the hind legs and the assistant on the right or left side, depending on the lesion. The endoscopist stayed near the head, on the left side. Laparoscopy was performed with five trocars, one each $11 \mathrm{~mm}$ and $10 \mathrm{~mm}$ and three that were $5 \mathrm{~mm}$. Trocar positioning depended on lesion location.

The intervention started with marking of the lesion border, both endoscopically and externally by laparoscopy ( $\mathbf{F i g . 2}$ ). The endoscopist then injected the $6 \%$ hydroxyethyl starch/indigo carmine staining solution around the tumor, thus highlighting the submucosa (blue color) and creating a buffer space between the mucosa and the seromuscular layer. This was important to prevent accidental opening of the mucosa by the surgeon.

With help of a hook, the surgeon incised the seromuscular layer around the lesion. As he dissected and pulled the submucosal block, the lesion remained attached to only the mucosa, which is quite elastic and redundant. Therefore, it was theoretically possible to either turn it in or to evert the lesion ( $\mathbf{F i g . 3}$ ).
Two traction sutures were secured on the extremities of the external seromuscular opening. A 5-mm trocar was inserted opposite the lesion. With a forceps, the lesion was pushed into the gastric lumen and the sutures were used for countertraction. At that moment, the endoscopist noticed the large "polyp," which was totally ensnared with a $3.0 \times 4.5-\mathrm{cm}$ hexagonal snare. The surgeon slowly removed his forceps so that the lesion, together with the seromuscular layer, remained fully grasped by the snare. As an important confirmation, he again checked whether the entire seromuscular layer was indeed caught by the endoscopic snare ( $\triangleright$ Fig. 4 ).

The surgeon started closing the seromuscular wound while the lesion was still being pulled by the endoscopist. Only afterwards the endoscopist applied a cutting current and the "tumor" was resected and removed through the mouth.

The endoscopist examined the resection bed and applied endoclips for secure closure ( $\mathbf{F i g}$.5). The gas injection leak test was performed and the specimen was examined to confirm that full-thickness gastric resection was complete and included the lesion as well as the mucosal/submucosal surrounding marks ( Fig. 6). 


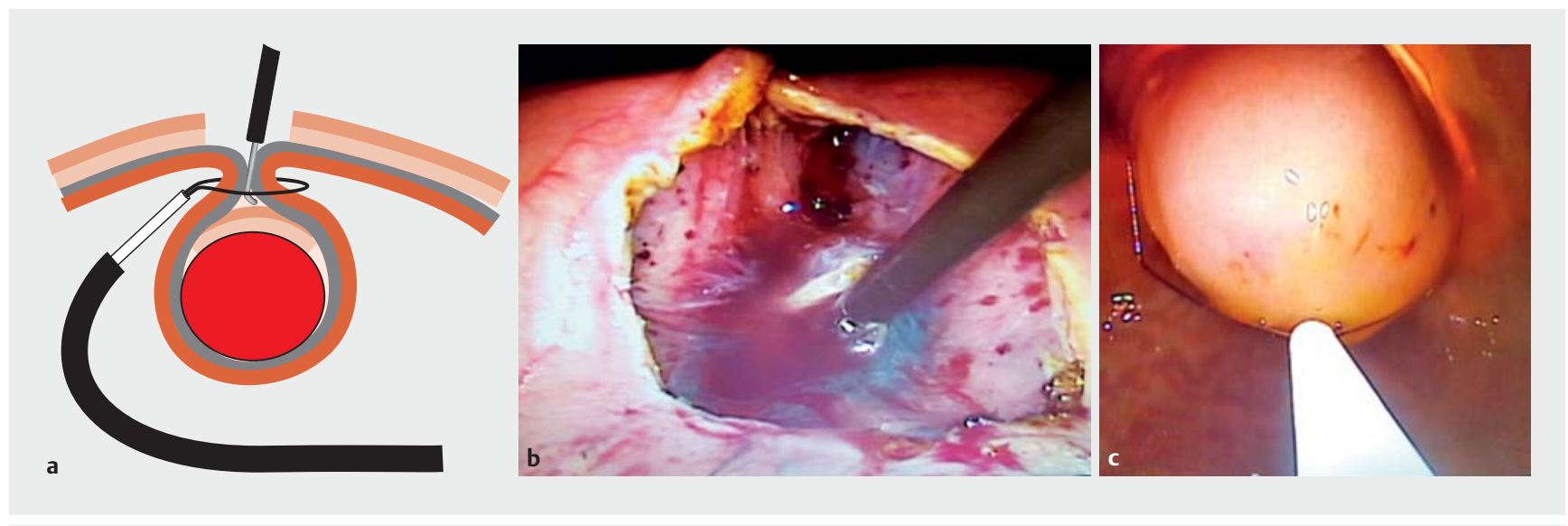

- Fig. 4 a Technical drawing of the invagination of the lesion and ensnaring of the block. b Laparoscopic view. $\mathbf{c}$ Endoscopic view.
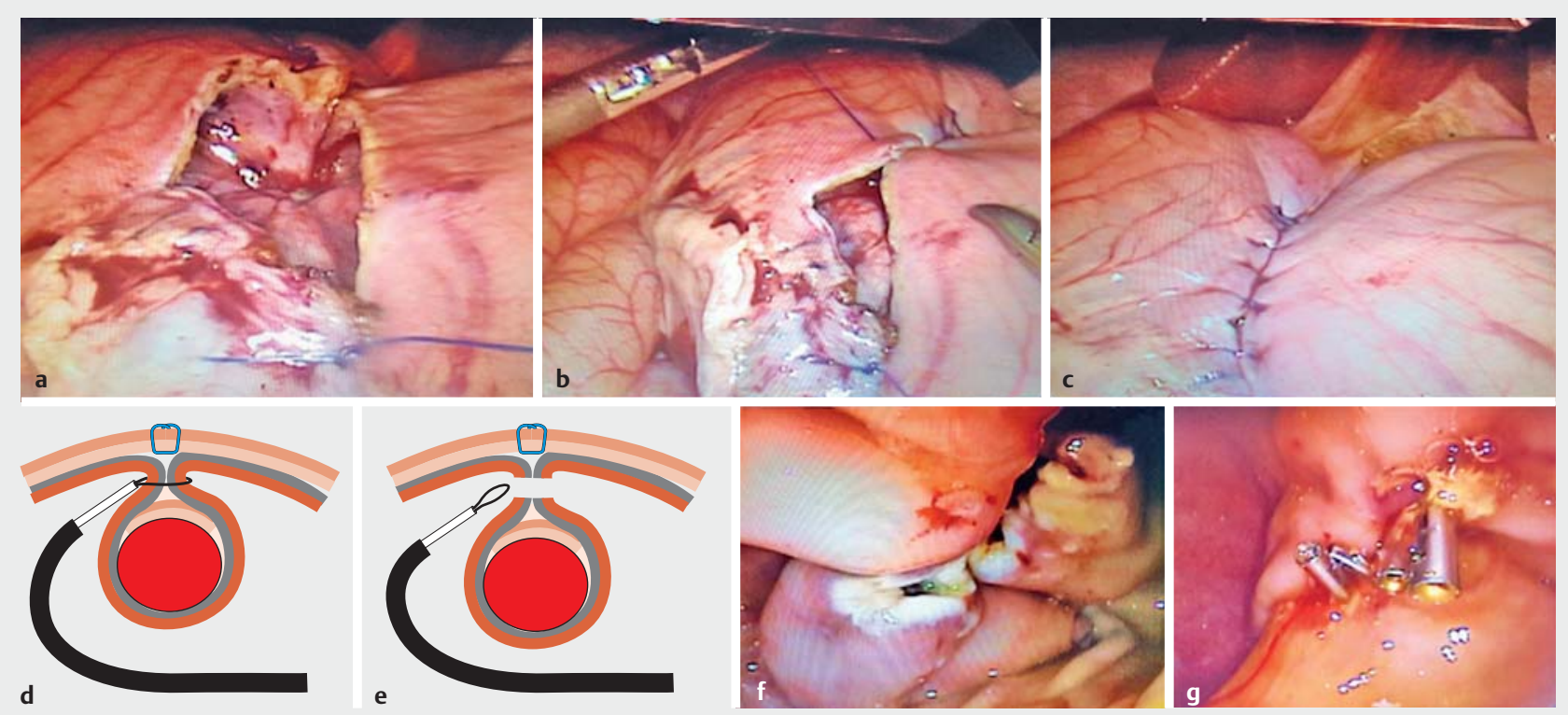

- Fig. 5 a Laparoscopic view of the lesion and seromuscular layer ensnaring. b Seromuscular layer closure. c Laparoscopic view of repaired seromuscular layer. $\mathbf{d}$, e Technical drawings of the procedure. $\mathbf{f}, \mathbf{g}$ Endoscopic view after removal of the lesion without and with endoclips.

\section{Assessment variables}

The primary endpoint was accomplishment of the procedure following all steps in the new technique, in the defined gastric locations. Duration was calculated from the beginning of the intervention until the gas injection leakage test. Margin examination was done only macroscopically and included confirmation that the "lesion" was surrounded by submucosa on all sides. Integrity of the mucosal covering at the luminal face and of the seromuscular layer at the peritoneal face was also mandatory to define successful full-thickness resection, with free margins. Perforation was visually checked and gas leakage was tested by means of gas injection.

\section{Results}

Seven pigs were us in our investigations, one for each location of a gastric lesion. All steps in the technique were successfully accomplished. Average duration of the procedure was $50 \mathrm{~min}$ utes (range 37 to 75 minutes). In all specimens the seromuscular layer was positively identified and the "tumor" was fully surrounded by mucosa, submucosa, and seromuscular tissue in macroscopic view. No mucosal perforation or gas leakage was identified. In such circumstances, primary and secondary aims alike were achieved. 


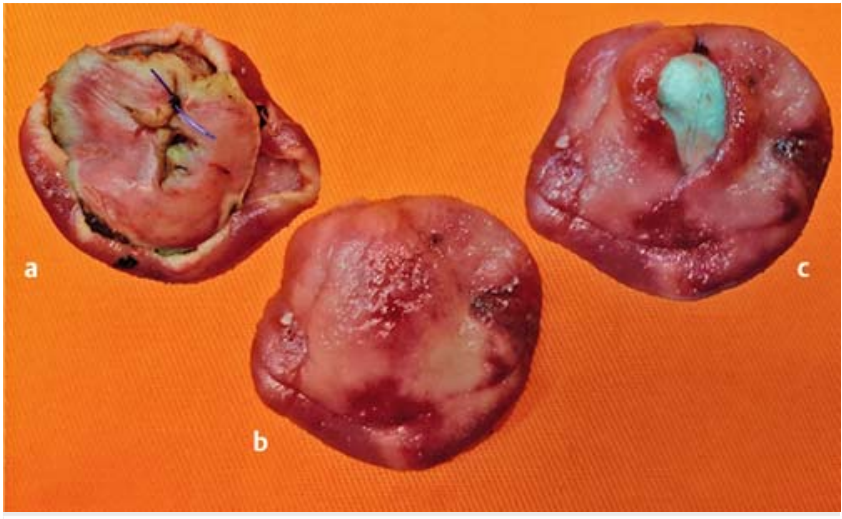

- Fig. 6 Specimen a Seromuscular side. b Mucosal side. c Opened mucosa, revealing the simulated tumor inside.

\section{Discussion}

Gastric lesions can be very heterogeneous with regard to size, location, and type of growth (intraluminal or extraluminal), among other features. With an arsenal of diversified techniques, the challenges inherent in each of these lesions can be handled in the best possible way.

Under the conditions in the current protocol, we demonstrated safe, precise, and effective full-thickness resection of gastric lesions. The technique was not very invasive nor timeconsuming and could be applied to tumors of $2 \mathrm{~cm}$ in various gastric locations. Because this was a pilot study, its reproducibility could not be evaluated, as the same endoscopist (PS) and surgeon (FHAM) conducted all the maneuvers.

Nonexposure modalities have been alluded to, such as a combination of laparoscopic and endoscopic approaches for treatment of neoplasia with a nonexposure technique (CLEANNET), and nonexposed endoscopic wall-inversion surgery (NEWS) [1,2]. With CLEAN-NET, the lesion is inverted away from the gastric mucosa after a seromuscular incision is made around the area. This technique appears ideal in cases of predominantly extraluminal lesions, which is different from our proposal. Like our procedure, NEWS involves invaginating the lesion towards the lumen, and thus it is appropriate for cases displaying luminal growth. In published experience with 20 cases, NEWS seemed safe and feasible [7].

The advantage of our intervention, with regard to NEWS, is that the endoscopist is not required to be skilled in endoscopic submucosal dissection (ESD). Although ESD is popular in Japan, it is not so familiar to Western endoscopists.

It must be admitted that when using a snare, it is sometimes impossible for the endoscopist to identify all marks around the lesion, thus increasing the risk of incomplete or piecemeal removal. That is why during laparoscopy, the surgeon needs to confirm whether the serosa up to the lesion is covered by the surrounding mucosa when it is ensnared ( $\mathbf{F i g}$.5a). A helpful detail concerning the mucosal side, which could be performed on larger lesions or in difficult locations, is application of endoclips to signal the limits of the snaring maneuver.
Maximum lesion size is a relevant concern, regarding not only the intrinsic limitations of the snaring resection, but also the ability to retrieve the mass through the mouth. In the series by Goto et al [7], a $35 \times 30 \mathrm{~mm}$ tumor was successfully extirpated, whereas Kikuchi et al [8] failed in a slightly larger case, faced with a diameter of $38 \mathrm{~mm}$. The option for larger masses is to cut the lesion after resection, performing piecemeal endoscopic removal, or alternatively, after incision of the gastric wall, laparoscopic removal.

According to Kikuchi et al [8], the NEWS technique could be improved (closed laparoscopic and endoscopic cooperative surgery/LECS) by initial endoscopic dissection of the submucosa, serosal marking, seromuscular suturing after the lesion was inverted towards the gastric lumen, and final endoscopic resection and exteriorization of the lesion. In a series of 10 patients, success was achieved in seven. One failure was attributed to the large size of the tumor, whereas inappropriate dissection was deemed responsible in the other two. Although different from our procedure in a number of details, that experience is consistent with the same principles.

Another technique described in the literature is hybrid pushpull endoscopic and laparoscopic full-thickness resection [9]. The seromuscular layer is not incised and the tumor is just pushed towards the gastric lumen by the surgeon, while the endoscopist tries to ensnare all the lesion and directly cut it. However, during this maneuver, there is a risk of gastric perforation and exposure of the lesion and the gastric contents to the peritoneal cavity. In four reported cases, the margins were compromised, and a complementary wedge resection was necessary, with use of a laparoscopic stapler [9].

The originality of our proposal consists of systematic and precise area marking and external incision, as well as a buffer zone separating the mucosa from the seromuscular layer, preceding inversion, ensnaring, defect closure, and resection. For subepithelial lesions of about $2 \mathrm{~cm}$, our experimental results were clearly successful. We speculate that even somewhat larger lesions could be addressed with the same approach, provided the growth is predominantly intraluminal.

There are reasons to believe that this technique would be convenient for subepithelial lesions in general, especially GIST and neuroendocrine tumors, not exceeding $35 \mathrm{~mm}$. Epithelial lesions (early gastric cancer) that do not require lymph node dissection would also be good candidates. Further studies targeting this modality are warranted because this study presents some limitations as it is a pilot study in an animal model, with a small sample size, performed in a single institution with the same operators and the long-term outcome cannot be assessed.

\section{Conclusions}

A safe and effective minimally invasive combined technique was described here. Expensive materials were not consumed, as ordinary surgical sutures, a snare and endoclips were the central tools. Lesion exposure, gastric content leakage, and incomplete resection were avoided. Technical difficulty was only moderate, mainly represented by optimal integration between 
the surgeons and the endoscopist. Given the favorable experimental results, human trials may be appropriate, with the aim of confirm feasibility and reproducibility of combined gastric full-thickness tumor resection in the clinical setting.

\section{Competing interests}

None

References

[1] Maehata T, Goto O, Takeuchi H et al. Cutting edge of endoscopic fullthickness resection for gastric tumor. World Gastrointest Endosc 2015; 7: $1208-1215$

[2] Kim HH, Uedo N. Combined laparo-endoscopic full-thickness resection techniques. Gastrointest Endoscopy Clin N Am 2016; 26: 335 373

[3] Hiki N, Yamamoto Y, Fukunaga T et al. Laparoscopic and endoscopic cooperative surgery for gastrointestinal stromal tumor dissection. Surg Endosc 2008; 22: 1729-1735
[4] Takeuchi H, Goto O, Yahagi N et al. Function-preserving gastrectomy based on the sentinela node concept in early gastric cancer. Gastric Cancer 2017; 20: S53-S59

[5] Komatsu S, Ichikawa D, Kosuga T et al. Clinical impact of laparoscopy and endoscopy cooperative surgery (LECS) on gastric submucosal tumor after its standardization. Anticancer Research 2016; 36: 3041 3048

[6] Goto O, Shimoda M, Sasaki M et al. Potential for peritoneal câncer cell seeding in endoscopic full-thickness resection for early gastric cancer. Gastrointest Endosc 2017; 87: 450-456

[7] Goto O, Takeuchi H, Sasaki M et al. Laparoscopy-assisted endoscopic full-thickness resection of gastric subepithelial tumors using a nonexposure technique. Endoscopy 2016; 48: 1010-1015

[8] Kikuchi S, Nishizaki M, Kuroda S et al. Nonexposure laparoscopic and endoscopic cooperative surgery (closed laparoscopic and endoscopic cooperative surgery) for gastric submucosal tumor. Gastric Cancer 2017; 20: 553-557

[9] Willingham FF, Reynolds P, Lewis M et al. Hybrid push-pull endoscopic and laparoscopic full thickness resection for the minimally invasive management of gastrointestinal stromal tumors: a pilot clinical study. Gastroenterology Reserchand Practice 2015; 2015: 1 - 7 